\title{
The Intestinal Microbiome of Viliuisk Encephalomyelitis Patients and Its Role in the Pathogenesis of the Disease
}

\author{
Tatyana Mikhailovna Sivtseva ${ }^{1 *}$, Galina Grigorievna Karganova ${ }^{2}$, \\ Vladimir Leonidovich Osakovskiy ${ }^{1}$
}

\author{
${ }^{I}$ North-Eastern Federal University, Research Center of the Medical Institute, 677027 Yakutsk, Republic of Sakha \\ (Yakutia), Russian Federation \\ ${ }^{2}$ Chumakov Federal Scientific Center for Research and Development of Immune-and-Biological Products of \\ Russian Academy of Sciences, 108819, Moscow, Russian Federation \\ *Corresponding author. Email: tm.sivtseva@s-vfu.ru
}

\begin{abstract}
Viliuisk encephalomyelitis (VE) is a unique endemic disease of the central nervous system, in which neurodegenerative processes are initiated by chronic aseptic subclinical inflammation of the brain. Recent research suggests that the gut microbiome play an important role in immune activation and inflammation in a variety of neurological conditions, including multiple sclerosis, Parkinson's disease, and Alzheimer's disease. The article discusses the results of a pilot study of the intestinal microbiome of healthy representatives of the Sakha ethnic group (Yakuts) and VE patients, carried out in collaboration with the Institute of Gene Biology of the Russian Academy of Sciences and the Atlas-Biomed Group - Knomics LLC. In the stool samples of VE patients the level of g.Prevotella, g.Faecalibacterium, f.Ruminococcaeae, as well as other unclassified members of o.Clostridiaceae was reduced. A significant increase in the proportion of bacteria g.Akkermansia, archaea Methanobrevibacter, and, on the verge of significance, of the families Christensenellaceae and Mogibacteriaceae was revealed. Analysis of the associations of the gut microbiome and the disease using the selbal algorithm showed a tendency for the association of VE with Methanobrevibacter, which is represented by M. smithii and one or more unclassified genera of the Coriobacteriaceae family. The revealed features of the taxonomic composition of intestinal microbiome in VE patients contribute to the development of a chronic subclinical inflammatory process, as well as pathological lipogenesis.
\end{abstract}

Keywords: intestinal microbiome, VE, encephalomyelitis, pathogenesis

\section{INTRODUCTION}

In Yakutia, among the socially significant agedependent neurodegenerative pathologies, the most famous are spinocerebellar ataxia (SCA1), amyotrophic sclerosis, Parkinson's and Alzheimer's diseases, as well as the unique endemic disease Viliuisk encephalomyelitis (in Yakut - bohooror (stiffness)). Viliuisk encephalomyeitis (VE) in terms of clinical-pathological and molecular-genetic characteristics is an interesting pathology, in which neurodegenerative processes are initiated by chronic aseptic subclinical inflammation of the brain. A typical picture of the clinical and pathological signes of the disease is pyramidal syndrome, brain tissue atrophy and edematous phenomena (spongiosis) of the brain, which brings VE etiologically to the group of spongy neurodegenerations $[1,2,3,4,5,6]$. A distinctive features of the VE pathogenesis are immunosuppression and neurodegeneration of the brain $[7,8]$. The results of the segregation analysis of patients with VE in the families of different foci of the disease show the coefficient of heritability for relatives of the first degree of kinship was $22-28 \%$. These data indicate that the contribution of the genetic component to the pathogenesis of the disease is $25 \%$, and the environmental factor is $75 \%$ [8]. A significant contribution of environmental factors does not exclude the influence of the genomic factor of the disease, its manifestation depends on the degree of modification variability of the genome. 
The main human organ that directly interacts with the factors of the external and internal environment of the body is the gastrointestinal tract. This is the habitat of a large population of microorganisms, while the specificity of the microflora is associated with the peculiarity of the ecological niche and biocenotic links with environmental factors. For the healthy functioning of the microbiome and the colon epithelium, it is necessary to maintain the nutrient medium and vitamins, which is carried out due to the products of fermentation by the symbiotic metabolism of the main microbial components forming the enterotype. The enterotype is formed through a long-term dietary habit, which reflects the response to the diet. In a healthy intestine, two to three main resistant intestinal clusters of microbes form, well-balanced symbiotic states between the microbiota and the human body. These are g.Bacteriodes, g.Prevotella (phylum Bacteriodetes) and g.Ruminococcus (phylum Firmicutes) [9, 10].

Recent studies indicate that the gut microbiota may play an important role in the activation of immunity and inflammation in various neurological conditions, including neurodegenerative pathologies, including multiple sclerosis, Parkinson's disease and Alzheimer's disease [11, 12, 13, 14, 15]. Numerous observations on the association of metabolic symbiosis of Christensenella and Methanobrevibacter with neurodegenerative pathologies (Parkinson's disease, Alzheimer's, multiple sclerosis, etc.) have been described and experimentally confirmed in model studies $[14,15]$. As studies have shown, the VE disease is no exception [16].

Veronika Kuznetsova, Alexandr Tyakht and their colleagues (Institute of Gene Biology, Russian Academy of Sciences and Atlas-Biomed Group Knomics LLC) for the first time carried out metagenomic studies of the intestinal microbiome of healthy representatives of the Sakha ethnic group (Yakuts) and VE patients [16]. In this paper we discuss the results of this pilot study.

\section{MATERIALS AND METHODS}

In the work, 11 stool samples from healthy people and 6 samples of patients with VE were analyzed. All patients were Yakuts. The study protocol was approved by the local committee on biomedical ethics of the "Yakut Scientific Center of Complex Medical Problems" of the Siberian Branch of the Russian Academy of Medical Sciences. Participants in the experiment filled out a questionnaire that included information about chronic diseases, medication intake, etc. Patients registered with a diagnosis of chronic VE underwent neurological examination.

The group of patients with chronic form of VE consisted of 3 males and 3 females aged from 48 to 80 years, on average 61 (standard deviation $(\mathrm{SD})=$ 11.6) years. The disease began at the age of 24 to 48 years, on average $32.3(\mathrm{SD}=7.5)$ years, in 4 patients with a meningoencephalitic phase. The duration of the disease is from 10 to $53(\mathrm{SD}=15.7)$ years. All patients had signs of the cranial nerves dysfunction and pyramidal signs, Babinski signs, 3 patients had severe dementia, 5 patients had dysarthria, muscle rigidity, muscle atrophy, bradykinesia of varying severity. Four patients underwent MRI and showed signs of severe cortical atrophy. Also, CSF was examined in 4 patients and the presence of intrathecal synthesis of oligoclonal IgG was revealed.

Control group without neurological symptoms consist of 4 males and 7 females, aged 26-60 years.

Stool samples were collected in sterile fecal collection tubes. After storage at $+4{ }^{\circ} \mathrm{C}$ for no more than 6 hours, the samples were placed at $-20{ }^{\circ} \mathrm{C}$. Further transportation was carried out with refrigerants without defrosting. After DNA isolation from the samples, the V3-V4 region of the $16 \mathrm{~S}$ rRNA gene was amplified. Sequencing was performed on an Illumina MiSeq platform. The number of reads was at least 20,000 per sample. Bioinformatic analysis was carried out in the Knomics-Biota system. The study design and methods are described in detail previously [16].

\section{RESULTS AND DISCUSSION}

The results of these studies are shown in Fig. 1. Taxonomic analysis of the 16S rRNA microbial gene reads obtained from all Yakut samples (healthy and VE patients) identified 75 genera in 44 families, which are grouped into two main symbiotic clusters: first included o. Clostridiales (Ruminococcaeae, Roseburia, Faecalibacterium and others); and another cluster - o.Bacteroideles (Parabacteroides, as well as Butyricimonas and Bilophila). Interesting is the presence of another original cluster of highly correlated microbes with low abundance, including Nesterenkonia, Halomonas, unclassified members of the Comamonadaceae, and others. 


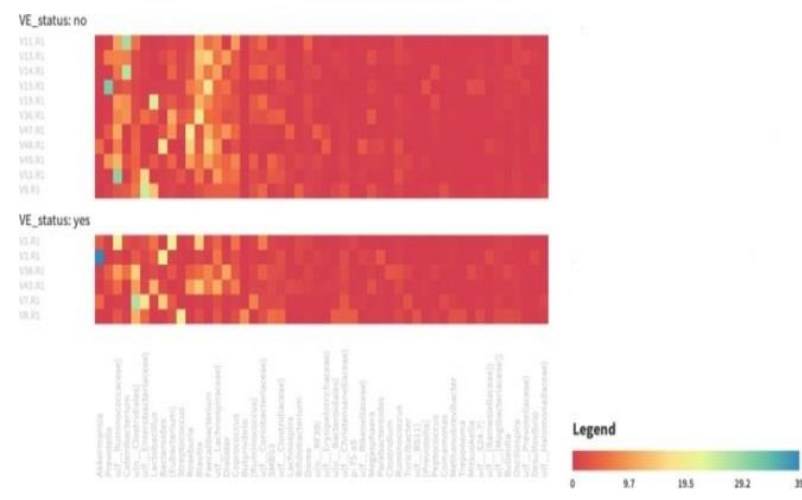

Fig.1. Gut community structure of Yakuts. Heatmap of the relative abundance of major microbial genera in the Yakut gut microbiome (snapshot of an interactive visualisation in the Knomics-Biota online system). The two subplots correspond to control subjects $(\mathrm{n}=11)$ and Viliuisk encephalomyelitis patients $(n=6)$. The bar plots on the top right show the levels of the ten most-abundant taxa averaged across all subjects [16]

VE disease significantly shifts the composition of the gut microbiome at different taxonomic levels. The main difference between the microbiome of VE patients is manifested at the taxonomic level of the genus (g). Despite the absence of significant differences in the alpha-diversity of microbiome bacteria in the samples of both groups, the differences are manifested by the relative abundance of bacteria in the microbiota of patients. In the stool samples of VE patients the level of g.Prevotella, g.Faecalibacterium, f.Ruminococcaeae, as well as other unclassified members of o.Clostridiaceae was reduced. A significant increase in the proportion of bacteria g.Akkermansia, archaea Methanobrevibacter, and, on the verge of significance, of the families Christensenellaceae and Mogibacteriaceae was revealed. Analysis of the associations of the gut microbiome and the disease using the selbal algorithm showed a tendency for the association of VE with Methanobrevibacter, which is represented by $M$. smithii and one or more unclassified genera of the Coriobacteriaceae family. Comparison of the results of metagenomic analysis of the microbiome of the VE patients with other neurodegenerative diseases such as Parkinson's disease and multiple sclerosis shows some common features, such as an increase in archaea Methanobrevibacter, Christensenellaceae and Akkermansia [9, 14, 15].

Studies indicate positive associations at the genus and species level between Christensenella spp. and Methanobrevibacter smithii, in which Christensenella spp. effectively supports the metabolism of $M$. smithii due to the competitive efficiency of the metabolic pathway of hydrogen production than Bacteroideles (B.thetaiotaomicron) and others [17]. Consumption of hydrogen by Methanobrevibacter smithii shifts the metabolic yield of the Christensenella minuta fermentation towards acetate rather than butyrate. The enterotype of the colon with this type of metabolism is phenotypically manifested by extreme manifestations of body mass index disorders (from anorexia to obesity), depending on ethnicity [18, 19, 20]. This data indicates that the gut enterotype with enrichment in Christensenella spp. and Methanobrevibacter smithii in the patient's microbiome is associated with pathological lipogenesis. At the same time, the disorders of body mass index depends on the genetics of the host of the carrier of a given microbiota [20, 21]. Pathological lipogenesis manifested in VE patients [8] is apparently associated with the enrichment of Christensenella spp. and Methanobrevibacter smithii in gut microbiota.

Studies of the gut microbiome of patients with multiple sclerosis show the enrichment of Akkermansia mucini, which is associated with the activation of pro-inflammatory pathways of the immune system [15]. Akkermansia (type Verrucomicrobia) - strict anaerobes, unlike other bacteria in the microbiome, use mucin as the only source of energy, carbon and nitrogen [23]. The glycoprotein mucin is a product of the epithelial layer of colon cells, which protects the epithelium [24]. Akkermansia play an important role in the decomposition of the upper loose mucus layer of the epithelium, producing oligosaccharides and short fatty acids (acetate, propionate). Enhanced acetate production and absorption contributes to the induction of pathogenetic lipogenesis. The proinflammatory features of Akkermansia may be associated with the destruction of mucus, contributing to an increase in the permeability of the epithelial layer and the availability of intestinal metabolites into the circulating blood. The penetration of intestinal metabolites into the blood allows interacting not only with receptors of intestinal epithelial cells, but also with immune blood cells. These receptors are widely expressed in immune and adipose cells, the activation of which induces a chronic subclinical inflammatory process and may trigger neurodegeneration $[25,26]$. As shown above, both of these processes are characteristic of the patient with VE.

\section{CONCLUSIONS}

A significant contribution of environmental factors ( $75 \%$ versus $25 \%$ of the contribution of the genetic 
component) to the development of VE disease may be a direct consequence of the activity of the intestinal microflora, which is directly related to the peculiarity of the ecological niche and biocenotic relationships with environmental factors. As the analysis of the intestinal microflora of the VE patients shows, the taxonomic composition of its microflora contributes to the development of a chronic subclinical inflammatory process, as well as pathological lipogenesis. It remains unclear what is the reason for the formation of a persistent chronic shift in the taxonomic composition of the gut microbiome in VE.

This work was supported by the Ministry of Science and Higher Education of the Russian Federation (Project FSRG-2020-0016 "Wide-genome studies of the gene pool of the indigenous population of the Arctic coast of Yakutia" 2020-2022 years).

\section{REFERENCES}

[1] P.A. Petrov, Neuropathology and Psychiatry, 6 (1958) 669-674

[2] A.N. Shapoval, Viliuisk encephalitis (1959)

[3] L.G. Goldfarb, D.C. Gaidusek, Brain, 115 (1992) 961-978

[4] T.M. Sivtseva, V.A. Vladimirtsev, R.S Nikitina, T.K. Davidova, D.A. Popov, V.L. Osakovsky, J. Neurol. Sci., 384 (2018) 84-88

[5] C.A. Mclean, C.L. Masters, V.A. Vladimirtsev, IA Prokhorova, LG Goldfarb, DM Asher, AI Vladimirtsev, VP Alekseev, DC. Gajdusek, Neuropathol. Appl. Neurobiology, 23 (1997) 212-217

[6] F.P. Avtsyn, A.A. Zhavoronkov, Archive of pathology, 56 (1994) 39-44

[7] V.L. Osakovsky, T.M. Sivtseva, Neuroimmunology, 10 (2012) 22-27

[8] L.G. Goldfarb, V.A. Vladimirtsev, N.M. Renvik, F.A. Platonov Vilyui encephalomyelitis. (SB RAS, 2014)

[9] M. Cheng, K. Ning, Genomics proteomics bioinformatics, 17 (2019) 4-12

[10] C. Liang, HC Tseng, HM Chen, et.al., BMC genomics,18 (supl.1) (2017) 932

[11] S. Gerhardt, MN. Mohajeri, Nutrients, 10 (6) (2018) 708

[12] Q. Ma, C Xing, W. Long, H.Y. Wang, Q. Liu, R.F. Wang, J. neuroinflammation, 16 (2019) 53
[13] V.A. Petrov, I.V. Saltykova, I.A. Zhukova, et all., Bull Exp Biol Med. 162(6) (2017) 734-737

[14] E.M. Hill-Burns, JW Debelius, JT Morton, et.al., Mov Disord., 32(5) (2017) 739749

[15] S. Jangi, R. Gandhi, LM Cox, et al., Nat Commun, 7 (2016) 12015

[16] V. Kuznetsova, A. Tyakht, L. Akhmadishina, et al., Orphanet J Rare Dis, 15 (2020) 327

[17] A. Ruaud, S. Esquivel-Elizondo, J. de la Cuesta-Zuluaga, et all., mBio, 11(1) (2020) e03235-19

[18] P.J. Turnbaugh, RE Ley, MA Mahowald, V Magrini, ER Mardis, JI.Gordon, Nature, 444 (2006) 1027-1031

[19] CA Mbakwa, J Penders, PH Savelkoul, C Thijs, PC Dagnelie, M Mommers, I. Arts, Obesity (Silver Spring), 23 (2015) 2508 -2516

[20] J.K. Goldrich, ER Davenport, M Beaumont, et al., Cell host microbe, 19 (2016) 731-743

[21] M. Beaumont, JK Goodrich, MA Jackson, et al., Genome Biol., 17(1) (2016) 189

[22] M.E. Johansson, M Phillipson, J Petersson, A Velcich, L Holm, GC Hansson, PNAS (USA), 105(39) (2008) 15064-15069

[23] M. Derren, EE Vaughan, CM Plugge, WM. de Vos, Int J Syst Evol Microbiol, 54 (2004) 1469-1476

[24] K.M. Tytgat, HA Büller, FJ Opdam, YS Kim, AW Einerhand, J. Dekker, Gastroenterology, 107 (1994) 1352-1363

[25] A. Nakajima, A. Nakatani, S. Hasegawa, PLoS One, 12(7) (2017) e0179696

[26] P.M. Smith, M.R. Howitt, N. Panikov, et.al., Science, 341(6145) (2013) 569-573 